\title{
THE STABILITY OF MASSIVE STARS
}

\author{
W. GLATZEL, M. KIRIAKIDIS and K.J. FRICKE \\ Universitäts-Sternwarte, Geismarlandstr. 11, D-37083 Göttingen, FRG
}

\section{Introduction}

An investigation of the stability properties of stellar models describing massive stars is motivated observationally by the necessity to explain the observed Humphreys - Davidson (HD) limit and the variability of the most massive stars known, i.e. the existence of luminous blue variables (LBVs). Theoretically, a determination of the upper mass limit for stable stellar objects together with its physical explanation and interpretation is of fundamental interest.

\section{Stability analysis}

The stability analysis is based on complete stellar models obtained from standard stellar evolution calculations with mass loss taken into account according to an empirical mass loss rate. Opacities were taken from the latest version of the OPAL library. Stability of stellar models is tested with respect to infinitesimal radial perturbations. For further details we refer the reader to Glatzel \& Kiriakidis (1993) and Kiriakidis et al. (1993).

Massive stars are found to suffer from strange mode instabilities with growth time scales in the dynamical range. Compared with these, instabilities driven by the the classical $\varepsilon$ and $\kappa$ mechanisms are negligible both with respect to range and strength. For solar chemical composition the domains of unstable stellar models in the HRD are shown in Fig.1.

Recently, Stothers \& Chin (1993, hereafter SC) have argued that the mean adiabatic exponent becoming smaller than $4 / 3$ is the origin of LBV instability. For their most unstable model (initial mass: $120 M_{\odot}$ ) we have redone their analysis using identical parameters. Concerning stellar evolution our object becomes significantly less massive and its effective temperature stays above $\log T_{\text {eff }}=3.83$ (lowest temperature given by SC: $\log T_{\text {eff }} \approx 3.65$ ). To cover the range of low temperatures reached in the calculations of SC we have also tested the stability of envelope models with appropriate masses and luminosities in this regime.

Contrary to SC both for the complete stellar models and the envelope models the mean adiabatic exponent always stays well above $4 / 3$. As $\left\langle\Gamma_{1}\right\rangle<$ $4 / 3$ is only a sufficient condition for instability, we have performed an adiabatic stability analysis of both complete stellar models and the envelope 
models. We find dynamical instability only below much lower effective temperatures $\left(\log T_{\text {eff }}<3.56\right)$ than those given by SC. Moreover, as evolutionary tracks do not reach sufficiently low temperatures these low critical temperatures can only be derived on the basis of envelope models. We emphasize that even the higher critical temperatures determined by SC would be too low to serve as an explanation for the LBV phenomenon or the existence of the HD limit.

HRD, $Z=0.02$

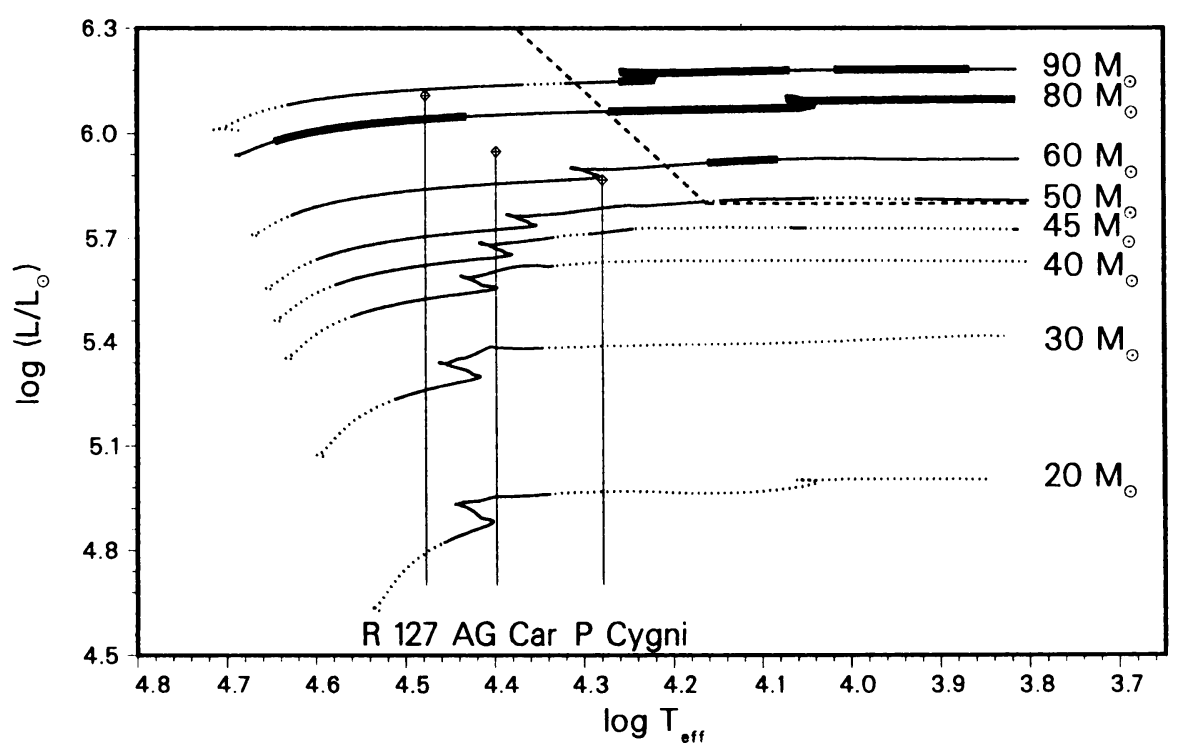

Fig. 1. HRD containing the evolutionary tracks of eight stars (dotted lines) with solar initial chemical composition and the initial masses indicated except for the $90 M_{\odot}$ track which corresponds to $(X, Y, Z)=(0.73,0.26,0.01)$. Unstable phases are denoted by solid lines, thick lines correspond to dynamical growth rates. Together with the observed positions of some LBVs the location of the HD limit is shown as a dashed line. The domain of instability covers the range of observed LBVs and extends to the main sequence for masses above $80 M_{\odot}$. For low masses the instability domain is continuously connected to the $\beta$ Cepheid instability strip. Note the agreement of the horizontal branch of the HD limit and the corresponding boundary of the instability domain. For high temperatures the instability domain is strongly metallicity dependent. It shrinks with decreasing metallicity and its blue edge ultimately becomes identical with the blue branch of the HD limit.

\section{References}

Glatzel, W., Kiriakidis, M.: 1993, MNRAS 263, 375

Kiriakidis, M., Fricke, K.J., Glatzel, W.: 1993, MNRAS 264, 50

Stothers, R.B., Chin, C.: 1993, ApJ 408, L85 